\title{
The lipid-laden foam cell: an elusive target for therapeutic intervention
}

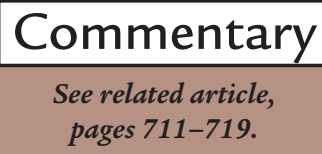

\author{
H. Bryan Brewer, Jr.
}

Molecular Disease Branch, National Heart, Lung, and Blood Institute, National Institutes of Health, Building 10, Room 7N115, 10 Center Drive MSC 1666, Bethesda, Maryland 20892-1666, USA.

Phone: (301) 496-5095; Fax: (301) 402-0190; E-mail: bryan@mdb.nhlbi.nih.gov.

Over the last decade, as our understanding of cellular cholesterol metabolism has advanced, a crucial role has emerged for acyl-coenzyme A:cholesterol-acyltransferase (ACAT) at several stages in the development of atherosclerosis. ACAT, the enzyme principally responsible for esterifying intracellular free cholesterol (FC) to cholesteryl esters (CEs), participates most directly in this process by promoting storage of lipid in arterial macrophages. This uptake is key to the disease process, as it transforms macrophage into arterial foam cells, the hallmark of atherosclerosis (1). In addition to its role in lipid loading of macrophages, ACAT also modulates cholesterol absorption from the intestine, secretion of VLDL from the liver, and steroidogenesis (2-5).

The FC that serves as a substrate for this enzyme may be derived directly from the diet or may be liberated from endocytosed lipoproteins by lysosomal hydrolases. The resulting CE can also be hydrolyzed back to FC in the cytoplasm by neutral CE hydrolase, and a cycle of cholesterol reesterification and hydrolysis ensues, providing both FC and CEs to satisfy the variable metabolic demands of different cells. ACAT's role in the reesterificationhydrolysis cycle is of particular importance in modulating the concentration of membrane FC, which, in excess, can be toxic to cells.

\section{Differential expression of ACAT genes}

The first breakthrough in our understanding of ACAT occurred in 1993 when T.Y. Chang and colleagues isolated and cloned a human ACAT cDNA by functional complementation of mutant $\mathrm{CHO}$ cells lacking ACAT activity (6). Recent studies indicate that the ACAT $4.3-\mathrm{kb}$ mRNA is synthesized from sequence encoded on 2 different chromosomes, 1 and 7, by a novel mechanism involving trans-splicing of 2 separate precursor RNAs (7). The mature ACAT enzyme is $69 \mathrm{kDa}$ and contains approximately 7 transmembrane regions (8). ACAT is a homotetrameric protein, which is regulated allosterically by cholesterol $(7,8)$; sterols also regulate ACAT at the translational level.

In 1996, Meiner et al. (9) reported the phenotype of the ACAT knockout mouse, which exhibits increased plasma total and HDL cholesterol levels but normal triglycerides and no reduction in the rate of intestinal cholesterol absorption. Cholesterol esterification is decreased in fibroblasts as well as adrenal membranes, and cholesteryl esters are markedly reduced in adrenal glands and peritoneal macrophages in the knockout animals. Of major importance, however, ACAT activity persisted in the liver of these knockout mice, providing the first clear indication that cholesterol esterification involves more that one ACAT enzyme. Consistent with this observation, Yang et al. (10) identified 2 separate enzymes in yeast that mediate sterol esterification.

Further clarification of the role of ACAT in cholesterol metabolism occurred in 1998, when 3 separate groups reported the cloning of a second ACAT gene, designated ACAT2 (11-13), which maps to chromosome 15 . The ACAT2 enzyme has little structural similarity to ACAT1 in its first approximately $100 \mathrm{NH}_{2}$-terminal amino acids, but the remainder of the sequences are nearly $60 \%$ identical (11). The tissue distribution of the 2 enzymes is of particular interest. ACAT1 is present in a wide variety of cells, including the macrophage, whereas ACAT2 is found primarily in the liver and intestine.

\section{ACAT1 and ACAT2 in normal and pathological lipoprotein metabolism}

A working model of lipoprotein metabolism is illustrated in Figure 1, highlighting the potential distinct functions of ACAT1 and ACAT2 in cholesterol absorption, intestinal and hepatic CE synthesis and transport of lipoproteins (1-5). In this model, ACAT2 is proposed to play a central role in cholesterol absorption from the intestine. Dietary CE, hydrolyzed to FC at least in part by pancreatic lipase, is taken up by enterocytes. In the enterocyte, $\mathrm{FC}$ is re-esterified to CEs by ACAT2, and the resulting CEs are incorporated with triglycerides into chylomicrons, which are then secreted from the cell. In the plasma, the triglycerides on chylomicrons are hydrolyzed by lipoprotein lipase (LPL), and the resulting chylomicron remnants, containing CE derived from enterocytes, are transported to the liver and removed from plasma by the hepatic LDL receptor and the LDL receptor-related protein (LRP). Chylomicron-derived CEs are hydrolyzed to FC in lysosomes, reesterified by hepatic ACAT2, packaged with triglycerides to form VLDLs, and secreted into the plasma. Triglycerides on VLDL are also hydrolyzed by LPL, and the lipoproteins are converted initially to IDLs and then to cholesterol-rich LDL. LDL may be removed from plasma in the liver and extrahepatic tissues by the LDL receptor or may undergo modification (e.g., oxidation) and be endocytosed by macrophages by means of the scavenger receptors CD36 and SR-A.

In this model, ACAT2 acts in the intestine and the liver, synthesizing $\mathrm{CE}$, which is then incorporated into lipoprotein particles and released into 
the plasma. ACAT1, on the other hand is widely distributed in tissues, and plays a pivotal role in cholesterol metabolism in steroidogenic tissues and macrophages. ACAT1-catalyzed CE synthesis in macrophages is of particular importance because these cells serve as the precursors to foam cells found in atherosclerotic lesions. When suitably modified LDL accumulates in the plasma, increased uptake of cholesterol by macrophages promotes foam cell formation and the development of atherosclerosis.

\section{Prospects for therapeutic use of ACAT inhibitors}

Knowledge of the critical role of ACAT in foam cell formation led to the development of numerous inhibitors of the enzyme. Several studies have shown that ACAT inhibitors prevent dietinduced atherosclerosis in hamsters and rabbits but typically lead to minimal changes in plasma cholesterol levels (14). Hence, these inhibitors may block disease progression by acting directly on the cells that form the atherosclerotic plaque (14); macrophage ACAT1 activity represents the most likely target of these drugs, as blockade of this activity would be predicted to interfere with foam cell formation and atherogenesis.

In the current issue of the JCI, Accad et al. crossed ACAT1 knockout mice with either ApoE knockout or LDL receptor (LDLR) knockout mice to test the prediction that abolishing ACAT1 activity would interfere with the development of atherosclerotic plaques (15). Surprisingly, in both hyperlipidemic models, ACAT1 deficiency caused extensive deposition of FC in the skin and brain. Using bone marrow transplantation, the authors established that ACAT1 deficiency in the macrophage alone was sufficient to cause dermal xanthomas in LDLR knockout mice. In addition, the ACAT1 knockout mice were still subject to atherosclerosis, although their atherosclerotic lesions contained markedly less lipid and fewer macrophages.

These elegant experiments establish the importance of ACAT 1 in 2 tissues, brain and skin, that have not received intensive study in the past.. The new finding of increased FC in the brain is

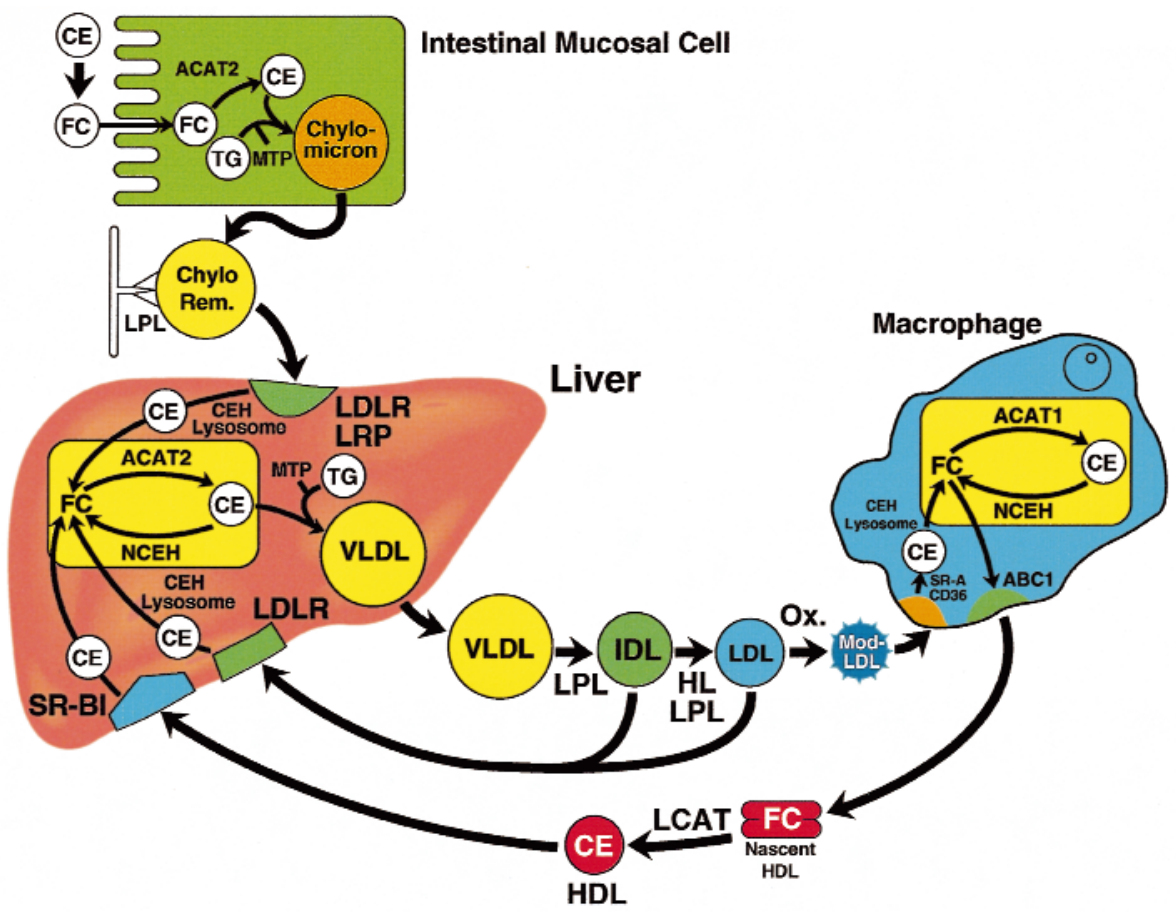

\section{Figure 1}

Schematic conceptual overview of cholesterol metabolism. Dietary CEs are converted to FC followed by absorption into the intestinal mucosal cell. In the enterocyte, FC is proposed to be esterified by CE by ACAT2, whereupon CE and triglycerides (TGs) are incorporated into chylomicrons. These particles are secreted initially into the lymph and then transferred to the plasma. The microsomal transfer protein (MTP) facilitates the transfer of lipids into lipoproteins. After secretion, the triglycerides on chylomicrons are hydrolyzed by LPL, and the lipid depleted CE-rich chylomicron remnant is taken up by the hepatic LDLR and the LRP. The CE on the chylomicron remnant is hydrolyzed by cholesteryl ester hydrolase (CEH) to FC in lysosomes. FC and CE can be readily interconverted by a reesterification-hydrolysis cycle: FC can be re-esterified to CE by ACAT2, and CE can be hydrolyzed to FC by a neutral cholesteryl ester hydrolase (NCEH). In the liver, CE and TG are incorporated into VLDLs and secreted into the plasma. VLDLs are initially converted to IDLs and then LDLs by LPL and hepatic lipase (HL). A portion of CE in IDL and LDL may be returned to the liver after interaction with the LDLR. LDL is modified (e.g., by oxidation), and the modified LDL is taken up by scavenger receptors, CD36 and SRA, in the macrophage. There, CE are hydrolyzed to FC in the lysosome and the FC may be converted to CE by ACAT1 and hydrolyzed by NCEH. Intracellular accumulation of CE converts a macrophage into a foam cell, the characteristic cell in atherosclerosis. Excess FC may be removed from the macrophage by apoA-I mediated FC efflux utilizing the $A B C 1$ transporter. In plasma FC on nascent HDL is converted to CE by lecithin cholesterol acyltransferase (LCAT) and the CE may be returned to the liver by selective uptake of the CE by the hepatic SR-B1 receptor. 
surprising and of great interest owing to the limited knowledge currently available on brain cholesterol metabolism. The consequences of increased FC in the brain, as well as the relative importance of defects in cholesterol metabolism in chronic brain disorders, deserve further study. Moreover, the accumulation of FC as skin xanthomas in ACAT1 knockout mice provides new insights into the important role of macrophage, and specifically, macrophage ACAT1, in cholesterol metabolism in the skin. This beneficial function of extravascular macrophages stands in contrast to pathological role of cholesterol uptake in foam cell in the vessel walls. With the discovery of 2 separate ACAT enzymes, it was anticipated that inhibitors of ACAT2 activity could be developed that would decrease cholesterol absorption, whereas specific ACAT1 inhibitors could be used to reduce foam cell formation and prevent atherosclerosis. The failure of ACAT1 deficiency in macrophages to block the development of atheroscle- rosis is particularly disappointing, but in light of the present results, the future development of specific ACAT1 inhibitors to prevent atherosclerosis must now be viewed with caution. The potential of ACAT 1 inhibitors to cause previously unsuspected side effects now requires further investigation.

1. Libby, P., et al. 1996. Macrophages and atherosclerosis plaque stability. Curr. Opin. Lipidol. 7:330-335.

2. Tabas, I. 1995. The stimulation of the cholesterol esterification pathway by atherogenic lipoproteins in macrophages. Curr. Opin. Lipidol. 6:260-268.

3. Chang, T.Y., Chang, C.C., and Cheng, D. 1997. Acyl-coenzyme A:cholesterol acyltransferase. Annu. Rev. Biochem. 66:613-638.

4. Dietschy, J.M. 1998. Dietary fatty acids and the regulation of plasma low density lipoprotein cholesterol concentrations. J. Nutr. 128 (Suppl.):444S-448S.

5. Joyce, C., Skinner, K., Anderson, R.A., and Rudel, L.L. 1999. Acyl-coenzyme A:cholesterol acyltransferase 2. Curr. Opin. Lipidol. 10:89-95.

6. Chang, C.C., Huh, H.Y., Cadigan, K.M., and Chang, T.Y. 1993. Molecular cloning and functional expression of human acyl-coenzyme A:cholesterol acyltransferase cDNA in mutant Chinese hamster ovary cells. J. Biol. Chem. 268:20747-20755.

7. Li, B.L., et al. 1999. Human acyl-CoA:cholesterol acyltransferase-1 (ACAT-1) gene organization and evidence that the 4.3-kilobase ACAT-1
mRNA is produced from two different chromosomes. J. Biol. Chem. 274:11060-11071.

8. Lin, S., Cheng, D., Liu, M.S., Chen, J., and Chang, T.Y. 1999. Human acyl-CoA:cholesterol acyltransferase- 1 in the endoplasmic reticulum contains seven transmembrane domains. J. Biol. Chem. 274:23276-23285

9. Meiner, V.L., et al. 1996. Disruption of the acyl$\mathrm{CoA}$ :cholesterol acyltransferase gene in mice: evidence suggesting multiple cholesterol esterification enzymes in mammals. Proc. Natl. Acad. Sci. USA. 93:14041-14046.

10. Yang, H., et al. 1996. Sterol esterification in yeast: a two-gene process. Science. 272:1353-1356.

11. Anderson, R.A., et al. 1998. Identification of a form of acyl-CoA:cholesterol acyltransferase specific to liver and intestine in nonhuman primates. J. Biol. Chem. 273:26747-26754.

12. Cases, S., et al. 1998. ACAT-2, a second mammalian acyl-CoA:cholesterol acyltransferase. Its cloning, expression, and characterization. J. Biol. Chem. 273:26755-26764

13. Oelkers, P., Behari, A., Cromley, D., Billheimer, J.T., and Sturley, S.L. 1998. Characterization of two human genes encoding acyl coenzyme A:cholesterol acyltransferase-related enzymes. J. Biol. Chem. 273:26765-26771.

14. Krause, R.R., and Bocan, T.M.A. 1995. ACAT inhibitors: physiologic mechanisms for hypolipidemic and anti-atherosclerotic activities in experimental animals. In Inflammation, mediators, and pathways. R.R. Ruffolo, Jr., and M.A. Hollinger, editors. CRC Press. Boca Raton, FL. 173-198.

15. Accad, M., et al. 2000. Massive xanthomatosis and altered composition of atherosclerotic lesions in hyperlipidemic mice lacking acyl CoA:cholesterol acyltransferase 1. J. Clin. Invest. 105:711-719 